\title{
Altered Oligodendroglial and Neuroglial Gene Expression in Adult Rat Cerebral White Matter Following Short- and Long-Term Ethanol Exposures and Abbreviated Abstinence
}

\author{
Yalcin $\mathbf{E B}^{1}$, Tong $\mathbf{M}^{1}$ and de la Monte $\mathbf{S M}^{2,3 *}$
}

${ }^{1}$ Liver Research Center, Division of Gastroenterology and Department of Medicine, Rhode Island Hospital and the Department of Pathology, Warren Alpert Medical School of Brown University, USA

${ }^{2}$ Departments of Neurology, Neurosurgery, and Pathology, Rhode Island Hospital and the Warren Alpert Medical School of Brown University, USA

${ }^{3}$ Department of Pathology \& Laboratory Medicine, Providence VA Medical Center, Providence, RI USA

Address correspondence to de la Monte SM, Suzanne_DeLaMonte_MD@Brown.edu

Received 03 October 2018; Revised 29 November 2018; Accepted 05 December 2018

Copyright (C) 2018 de la Monte SM, et al. This is an open access article distributed under the terms of the Creative Commons Attribution License, which permits unrestricted use, distribution, and reproduction in any medium, provided the original work is properly cited.

\begin{abstract}
Ethanol-mediated white matter degeneration is associated with impaired oligodendrocyte functions linked to myelin maintenance. This study examines ethanol-induced time course-dependent shifts in myelin gene expression in a chronic + binge exposure model and assesses therapeutic responses to short-term abstinence. Six-week-old male Long Evans rats were fed isocaloric liquid diets with $0 \%$ or $26 \%$ ethanol (caloric content) for 3 or 8 weeks. During the last 2 weeks of ethanol feeding, the rats were binged with $2 \mathrm{~g} / \mathrm{kg}$ ethanol by intraperitoneal injection, $3 \mathrm{x} /$ week. In a subgroup pair-fed for 6 weeks, ethanol was tapered over 2 days and then withdrawn for 12 days. Gene expression in temporal lobe white matter was measured by qRT-PCR analysis. Chronic+binge ethanol exposures caused short-term, long-term, or step-wise changes in neuroglial gene expression such that: 1) NCAM1 mRNA levels were significantly elevated after 3 weeks $(P=0.04)$; 2) PLP was reduced after 8 weeks $(P=0.003)$; and 3$)$ MAG $(P=0.008)$ and RTN4 $(P=0.02)$ progressively declined from 3 to 8 weeks of exposure. Abstinence did not reverse long-term ethanol effects on PLP, MAG or RTN4. Instead, it significantly reduced RPAIN $(P=0.002)$ and Olig2 $(P=0.02)$ relative to responses after 8 weeks of continuous ethanol exposure. In conclusion, chronic+binge ethanol exposures mainly inhibited mature myelin-associated gene expression. Short-term abstinence failed to abrogate those adverse effects of ethanol. Instead, the corresponding reductions in RPAIN and Olig2 expression suggest that during the acute phase of withdrawal or abstinence, molecular indices of white matter degeneration may worsen and that additional therapeutic measures may be required for optimum recovery.
\end{abstract}

\section{Keywords}

chronic+binge alcohol; short-term abstinence; myelin; white matter degeneration

Abbreviations: CNS: central nervous system; PDGFR- $\alpha$ : plateletderived growth factor receptor-alpha; GALC: galactosylceramidase; GC: group-specific component vitamin D binding protein; PROM1: prominin 1; MBP: myelin basic protein; PLP: proteolipid protein; MAG: myelin-associated glycoprotein; MOG: myelin oligodendrocyte glycoprotein; RTN4: reticulon 4; RPAIN: RPA interacting protein; ST8Sia1: ST8 alpha-N-acetyl neuraminide alpha-2,8-sialytransferase 1; NCAM1: neural cell adhesion molecule 1; GSTP-1: glutathione S-transferase P-1; CSPG4: chondroitin sulfate proteoglycan 4; GFAP: glial fibrillary acidic protein; NTRK2: neurotrophic tyrosine kinase receptor, Type 2; FOXO1: forkhead Box O1; FOXO4: forkhead Box O4; Olig1: oligodendrocyte transcription factor 1; Olig2: oligodendrocyte transcription factor 2; NKX6-1: NK6 homeobox 1; PAX6: paired box 6; qRT-PCR: Quantitative Reverse Transcriptase Polymerase Chain Reaction; HPRT: hypoxanthine-guanine phosphoribosyltransferase; Act $\beta$ : beta-actin; ANOVA: analysis of variance

\section{Introduction}

Heavy drinking over a long period of time adversely affects the central nervous system (CNS), impairing cognition and memory and causing neurodegeneration in adults $[1,2]$. Pathological and neuroimaging studies have shown that one of the main neurodegenerative effects of chronic alcohol exposure is white matter atrophy [35]. Experimental models revealed that the underlying pathologies of alcohol-induced white matter atrophy include demyelination, dysmyelination, and axonal degeneration [6]. Within the cerebrum, alcohol's targeting of the corpus callosum has been studied extensively due to corresponding deficits in inter-hemispheric communication linked to cognitive and motor deficits [2]. Magnetic resonance imaging (MRI) and postmortem studies have further demonstrated that alcohol-mediated callosal thinning worsens with advancing age [7,8], yet, with sustained abstinence, partial recovery of white matter damage $[9,10]$ and cognitive function $[11]$ can be achieved in adults. 
In the CNS, oligodendrocytes generate myelin which is needed to ensure structural and functional integrity of axons. Although myelination occurs throughout life $[12,13]$, the largest increases occur during the early stages of development and continue through adolescence. In humans, CNS myelination is most robust during the first two decades of life [14] whereas in rodents, myelination occurs mainly during first two postnatal months [15]. Myelin is largely composed of lipids (70\%), but also contains structural proteins [16]. The compositions of oligodendrocyte myelin lipids and proteins change with white matter maturation during development [17]. Myelin-associated proteins expressed by immature oligodendrocytes include nestin, vimentin, chondroitin sulfate proteoglycan 4 (CSPG4), platelet derived growth factor receptor ( $\alpha$-subunit, PDGFR- $\alpha$ ), galactocerebroside/galactoceramidase (GALC), and prominin 1 (PROM1) [18, 19], whereas those expressed by mature oligodendrocytes include myelin basic protein (MBP), myelin-associated glycoprotein (MAG), myelin oligodendrocyte glycoprotein (MOG), proteolipid protein (PLP), glutathione S-transferase P-1 (GSTP-1), and galactocerebroside/galactoceramidase (GALC) [1921].

High-level binge ethanol exposures $(5 \mathrm{~g} / \mathrm{kg}$, oral gavage) have devastating effects on both neurons and oligodendrocytes, impairing proliferation and differentiation [22]. Repeated binge ethanol exposures $(9.3 \mathrm{~g} / \mathrm{kg} /$ day infused over 4 days $)$ decrease survival of oligodendrocyte progenitor cells in adult rat brains [22]. Chronic (26\% caloric ethanol for 8 weeks) plus binge (2 $\mathrm{g} / \mathrm{kg}$ ethanol by intraperitoneal injection, 3 times/week during weeks 7 and 8) exposures inhibit expression of mature oligodendrocyte myelin-associated proteins and de novo synthesis of myelin in adult rats [23,24]. Furthermore, ultrastructural studies showed that in the chronic+binge rat model which mimics consumption patterns in humans with alcohol use disorders, frontal lobe white matter injury is due to combined pathologies in myelin (demyelination and dysmyelination) and axons (axonal degeneration) [6]. Similar observations have been made in postmortem human alcoholic brains [25]. However, abstinence following alcohol exposure can result in increased cell proliferation and formation of new neurons and oligodendrocytes, reflecting neurogenesis and myelination in adults $[26,27]$.

Previous reports demonstrating the potential for at least partial recovery of white matter atrophy and degeneration in humans and experimental models $[5,9,28]$, along with improvements in cognitive-executive functions in humans following periods of abstinence [11], justifies further investigation to determine the mechanisms of those therapeutic responses. To begin addressing this question, the present work utilized an adult rat chronic+binge ethanol exposure model to examine time course-dependent shifts in myelin-associated gene expression and assess therapeutic responses to short- term abstinence. Our working hypothesis was that the progressive white matter atrophy and degeneration caused by chronic + binge ethanol exposures is mediated by impairments in the expression of myelin-associated proteins and transcription factors that are essential to myelin and axon generation, maturation, and maintenance. In addition, we proposed that the partial restoration of white matter integrity following short-term abstinence would be associated with increased expression of mature versus immature myelin-associated proteins. This work is novel because it investigates the molecular pathogenesis of alcohol-mediated white matter degeneration over time and in relation to abstinence.

\section{Methods}

\subsection{Experimental model}

Experiments using rats were conducted in accordance with the Guide for the Care and Use of Laboratory Animals as adopted and promulgated by the U.S. National Institutes of Health, and approved by the Lifespan Institutional Animal Care and Use Committee. Six-weekold male Long Evans rats were fed with isocaloric liquid diets containing $0 \%$ or $26 \%$ ethanol by caloric content for 3 or 8 weeks ( $n=6 /$ group). Ethanol-fed rats were administered intraperitoneal injections of $2 \mathrm{~g} / \mathrm{kg}$ ethanol 3 times per week during the last 2 weeks of ethanol feeding; controls were treated with saline. A subgroup $(n=6)$ exposed to 6 weeks of ethanol was subjected to a brief period of abstinence by tapering the ethanol over 2 days, and then maintaining them on ethanol-free diets for 12 days. Since neurodegenerative effects of alcohol are detectable by 4-6 weeks of chronic ethanol feeding, we initiated abstinence after 6 weeks of ethanol exposure when the deleterious effects of ethanol would have been manifested. The short period of abstinence was used because in previous studies, a two-week interval was sufficient to increase neurogenesis [29] and improve spatial learning and memory [30] in adults.

On the day of sacrifice, body weights, brain weights, and blood alcohol concentrations were measured (Supplementary Figure 1). Upon sacrifice, the temporal lobes were harvested, frozen on dry ice, and stored at $-80^{\circ} \mathrm{C}$. Although the neurotoxic and degenerative effects of alcohol occur prominently in the prefrontal cortex, temporal lobe, hippocampus, and cerebral white matter [2], our studies focused on its adverse effects in temporal white matter as an extension of previous work using this model in which we demonstrated alcohol-induced temporal lobe white matter atrophy with myelin loss, aberrant expression of mature glial and neuronal proteins, and sphingolipid and phospholipid profiles [23,28].

2.2. Targeted Quantitative Reverse Transcriptase Polymerase Chain Reaction (qRT-PCR) Arrays

Total RNA extracted from fresh frozen tissue using the RNeasy Mini Kit (Qiagen, Hilden, Germany) was reverse transcribed using the AMV 1st Strand cDNA 
Synthesis Kit (Roche, Indianapolis, IL). The resulting cDNA templates were used to measure mRNA transcripts encoding 18 neuroglial and 6 transcription factor genes by qPCR in a Roche Lightcycler 480 System [23]. Primer pairs were designed using Primer 3 software (https://www.ncbi.nlm.nih.gov/tools/primer-blast/). Relative mRNA abundance was calculated using the $2^{-\Delta \Delta \mathrm{Ct}}$ method with results normalized to internal controls (Hypoxanthine-guanine phosphoribosyltransferase (HPRT) and Beta-actin (Act $\beta)$ ).

\subsection{Statistical analysis}

Results are presented as mean \pm standard deviation. Inter-group comparisons were made by one-way or twoway analysis of variance (ANOVA) with Tukey post hoc tests (GraphPad Prism 6, San Diego, CA, USA). Each experimental group included 6 rats. Post hoc test significant differences $(\mathrm{P} \leq 0.05)$ and trend effects $(0.05$ $\leq \mathrm{P} \leq 0.1)$ are shown in the graphs.

\section{Results}

\subsection{Glial neuronal markers investigated}

We used a custom targeted PCR array to measure mRNA transcripts encoding structural and transcription factor proteins expressed in immature or mature oligodendroglia. The rationale for selecting the gene targets was based on known shifts in molecular markers of oligodendroglia that occur with maturation. Oligodendrocytes arise from oligodendrocyte precursor cells (OPCs) that express high levels of nestin, vimentin, chondroitin sulfate proteoglycan 4 (CSPG4), platelet derived growth factor receptor ( $\alpha$-subunit, PDGFR$\alpha$ ), galactocerebroside/galactoceramidase (GALC), and prominin 1 (PROM1) [18, 19]. Differentiation of OPCs into immature oligodendrocytes are regulated by transcription factors Olig1, Olig2, NKX6-1, and PAX6 $[18,31]$. Immature oligodendrocytes progress into mature oligodendrocytes that contain myelin specific proteins such as myelin basic protein (MBP), myelin associated glycoprotein (MAG), myelin oligodendrocyte glycoprotein (MOG), proteolipid protein (PLP), and glutathione S-transferase P-1 (GSTP-1) [19-21]. In addition, reticulon 4 (RTN4), RPA interacting protein (RPAIN), and ST8 alpha-N-acetyl neuraminide alpha2,8-sialytransferase 1 (ST8Sia1) have been used as a marker of oligodendrocytes and myelin sheaths [3133]. NCAM 1 and NTRK 2 are neuronal markers that mediate neuronal development, survival, proliferation, differentiation, and plasticity [34, 35]. GFAP is an intermediate filament protein used as astrocyte marker. FOXO-1 and FOXO-4 are transcription factors that target insulin signaling and regulate oligodendrocyte precursor development and myelination [36, 37].

3.1.1. Short- versus long-term ethanol exposure and abbreviated abstinence effects on immature oligodendroglial gene expression: For immature oligodendroglia we measured mRNA levels of nestin, vimentin, platelet-derived growth factor receptor-alpha (PDGFR- $\alpha$ ), galactosylceramidase (GALC), group-specific component vitamin D binding protein (GC), and prominin 1 (PROM1). Two-way ANOVA tests demonstrated ethanol exposure $\mathrm{x}$ time interactive trend effects for Nestin $(P=0.08)$ and a trend effect for duration of ethanol exposure on GC expression $(P=0.07)$. In addition, one-way ANOVA with Tukey multiple comparisons of 8-week control, 8-week ethanol, and abstinence groups demonstrated that short-term abstinence had no significant or trend effects on immature oligodendroglia.

3.1.2. Short- versus long-term ethanol exposure and abbreviated abstinence effects on mature oligodendroglial gene expression: For mature myelinating oligodendroglia, we measured myelin basic protein (MBP), proteolipid protein 1 (PLP-1), myelin-associated glycoprotein 1 (MAG-1), myelin oligodendrocyte glycoprotein 1 (MOG-1), reticulon 4 (RTN4), RPA interacting protein (RPAIN), and ST8 alpha-N-acetyl neuraminide alpha2,8-sialytransferase 1 (ST8Sia1). Two-way ANOVA tests demonstrated that the duration of ethanol exposure had significant inhibitory effects on MAG $(P=0.008)$ and RTN4 $(P=0.02)$ and a trend effect on RPAIN $(P=0.07)$ expression. In addition, significant ethanol and time interactive effects were observed for PLP and MOG expression $(P=0.04)$. Long term ( 8 weeks) ethanol exposure significantly reduced PLP expression $(P=0.003)$ and had a trend decrease in MOG expression $(P=0.07)$ relative to controls (Figures $1 \mathrm{~A}$ and $1 \mathrm{C})$. Also, it is noteworthy that the mean levels of MBP expression increased with age and decreased with ethanol exposure over the course of the experiment. However, the intergroup statistical differences failed to reach significance due to the large standard deviations (Figure 1E). Shortterm abstinence failed to normalize ethanol mediated reductions in PLP and MOG expression and decreased RPAIN expression relative to control $(P=0.002)$ and ethanol groups $(P=0.004)$ (Figures $1 \mathrm{~A}, 1 \mathrm{C}$, and 1D). ST8Sial expression was similar in control and ethanol brains over time and remained unaltered with short-term abstinence (Figure 1G).

3.1.3. Short- versus long-term ethanol exposure and abbreviated abstinence effects on neuroglial gene expression: For neuroglial markers, we measured mRNA levels of neural cell adhesion molecule 1 (NCAM1), glutathione S-transferase P-1 (GSTP1), chondroitin sulfate proteoglycan 4 (CSPG4), glial fibrillary acidic protein (GFAP), and neurotrophic tyrosine kinase receptor, Type 2 (NTRK2). Ethanol exposure had significant stimulatory effects on NCAM1 expression at the 3 -week time point $(P=0.04)$, while mRNA expression remained unchanged in control, ethanol, and abstinence groups at the 8-week time point (Figure 2A). Furthermore, two-way ANOVA tests revealed significant ethanol $\mathrm{x}$ time interactive effects $(P=0.04)$ and a trend effect for ethanol exposure $(P=0.06)$. The mean levels of GSTP1 and NTRK2 decreased with ethanol exposure 

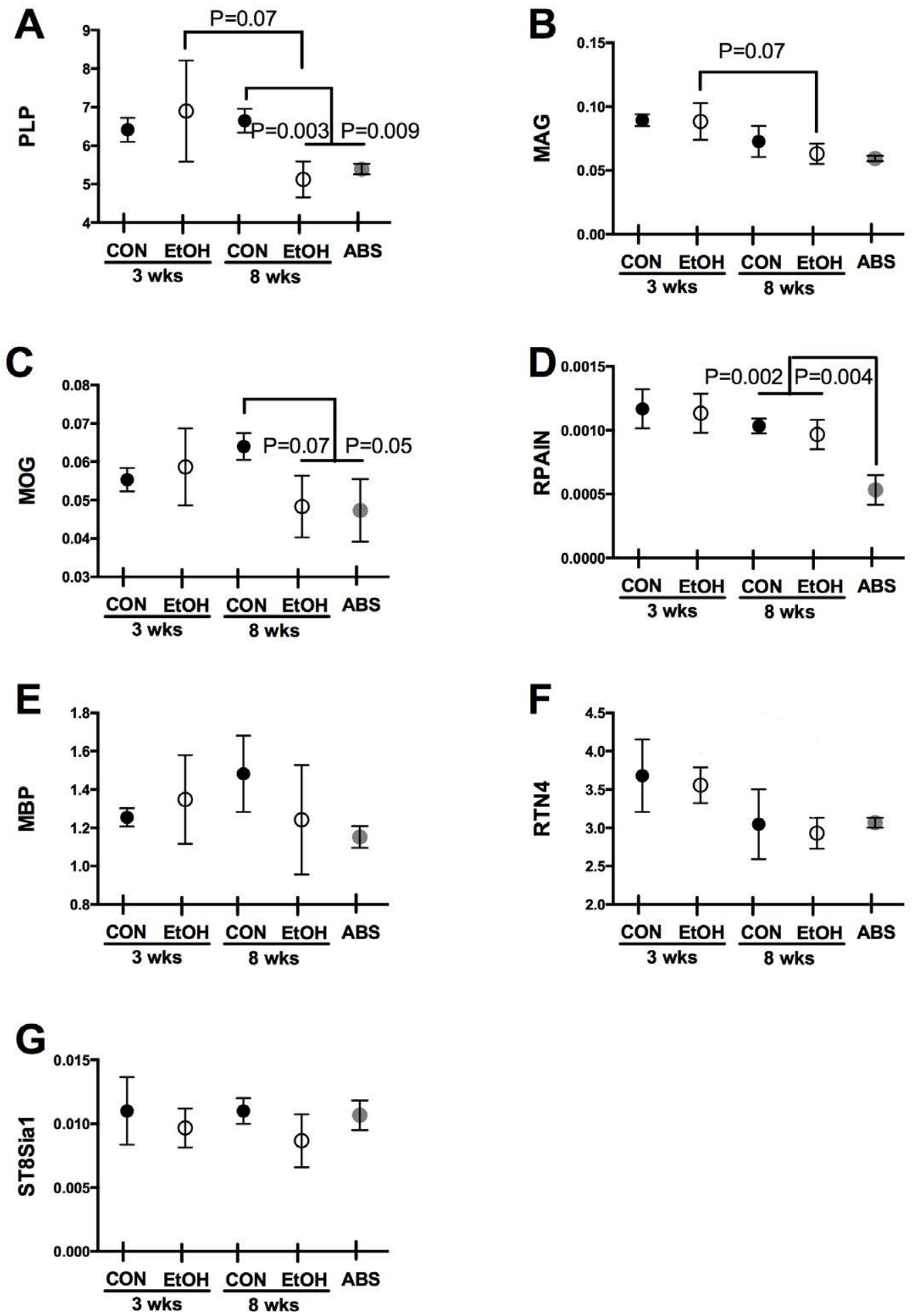

Figure 1: Short- versus long-term ethanol exposure and abbreviated abstinence effects on mature oligodendroglial gene expression. RNA extracted from control, ethanol-exposed, and ethanol-abstinent temporal lobes were reverse transcribed, and cDNAs were used to measure oligodendroglial gene expression by qPCR analysis. Gene expression was calculated by the $2^{-\Delta \Delta C t}$ method and results were normalized to Hypoxanthine-guanine phosphoribosyltransferase (HPRT) and Beta-actin (Act $\beta$ ). Graphs represent mRNA levels for (A) PLP, (B) MAG, (C) MOG, (D) RPAIN, (E) MBP, (F) RTN4, and (G) ST8Sia1. Inter-group comparisons were made by one way and two-way analysis of variance (ANOVA) with Tukey post hoc tests.

and time; however, the statistical analysis failed to reach significance due to large variations within the group (Figures $2 \mathrm{~B}$ and 2C). Ethanol or abstinence had no significant or trend effects on CSPG4 and GFAP expression (Figures 2D and 2E).
3.1.4. Short- versus long-term ethanol exposure and abbreviated abstinence effects on transcription factor gene expression: Glial transcription factors include Forkhead Box O1 and $\mathrm{O} 4$ (FOXO1, FOXO4), oligodendrocyte transcription factor 1 and 2 (Olig1, 

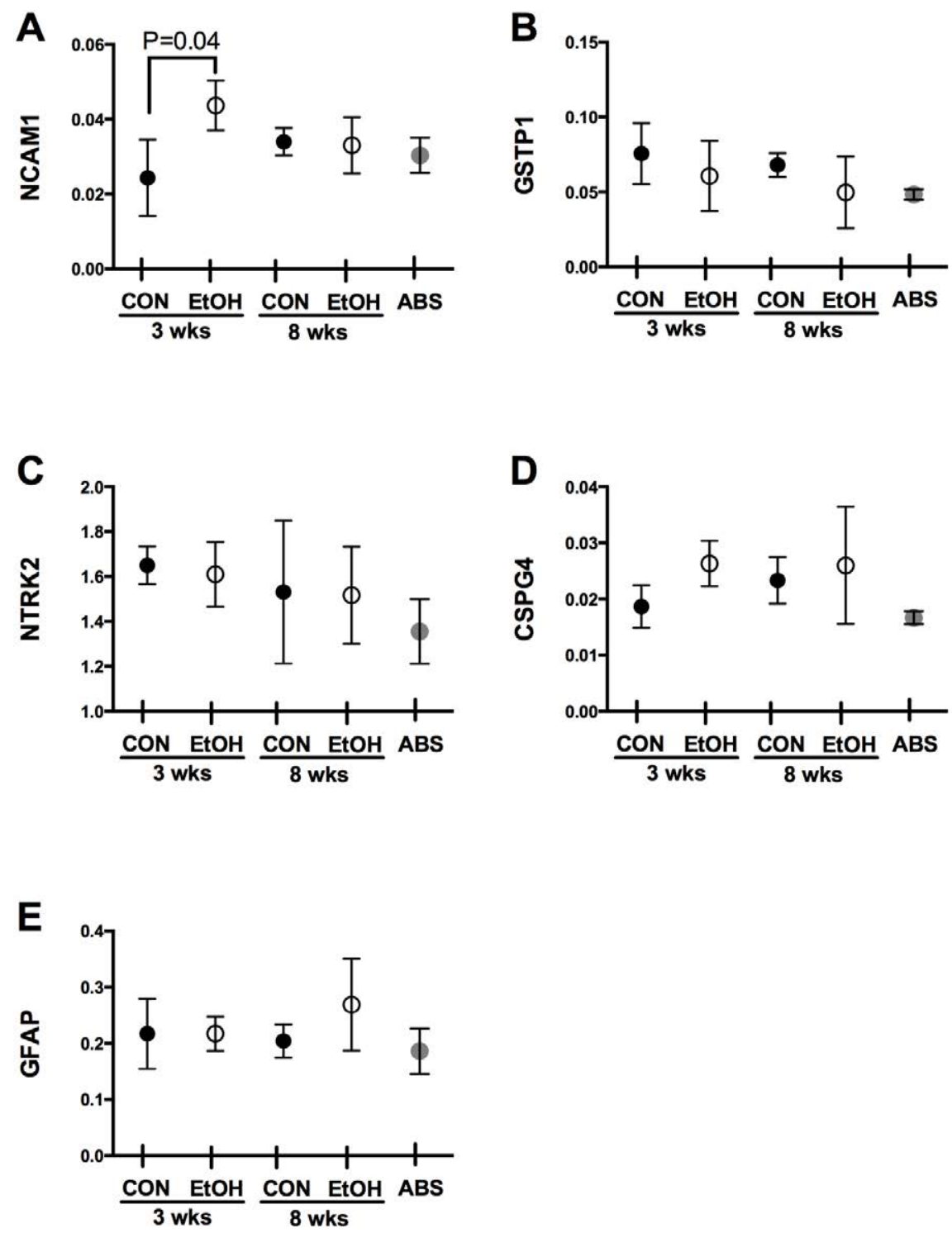

Figure 2: Short- versus long-term ethanol exposure and abbreviated abstinence effects on neuroglial gene expression. mRNA levels of (A) NCAM1, (B) GSTP1, (C) NTRK2, (D) CSPG4, and, (E) GFAP were measured in temporal lobes of control, ethanol-exposed, and ethanol-abstinent rats by qPCR analysis. Gene expression was calculated by the $2^{-\Delta \Delta C t}$ method and results were normalized to Hypoxanthine-guanine phosphoribosyltransferase (HPRT) and Beta-actin (Act $\beta$ ). Inter-group comparisons were made by one-way and two-way analysis of variance (ANOVA) with Tukey post hoc tests.

Olig2), NK6 Homeobox 1 (NKX6-1), and Paired box 6 (PAX6). FOXO1 expression was increased trend-wise by ethanol exposures at the 3 -week time point relative to control brains $(P=0.1)$. However, expression reduced trend-wise over time in the ethanol group and remained at low levels after abstinence $(P=0.1)$ (Figure 3A). Two-way ANOVA tests detected significant ethanol $\mathrm{x}$ time interactive effects for FOXO1 expression in the temporal lobe $(P=0.01)$. FOXO4 was similarly expressed in control, ethanol, and abstinence groups and remained unaltered over time (Figure 3B). Significant stimulatory ethanol exposure $(P=0.02)$ and duration effects $(P=0.05)$ were observed for Olig2 expression. In contrast, shortterm abstinence significantly decreased Olig2 mRNA levels relative to 8 -week ethanol exposed brains $(P=0.01)$ (Figure 3D). A similar expression pattern was observed for Olig1 in control and ethanol samples, but the statistical analysis failed due to a large variation in the 8-week ethanol exposed group (Figure 3C). NKX6-1 and PAX6 expression remained unchanged after shortor long-term ethanol exposures or abbreviated abstinence (Figures 3E and 3F).

\section{Discussion}

Herein, we examined time-dependenteffects of chronic + binge ethanol exposures and short-term abstinence on white matter myelin, neuroglial, and transcription factor gene expression in the temporal lobes of adult male Long Evans rats. The chronic ethanol exposures were initiated during late adolescence (6 weeks of age) and binging was performed when the rats were adults at either 8 to 9 weeks (short-term exposure) or 11 to 14 weeks (longterm exposure) of age. Ethanol abstinence began after 6 weeks of chronic and 2 weeks of binge ethanol exposures 

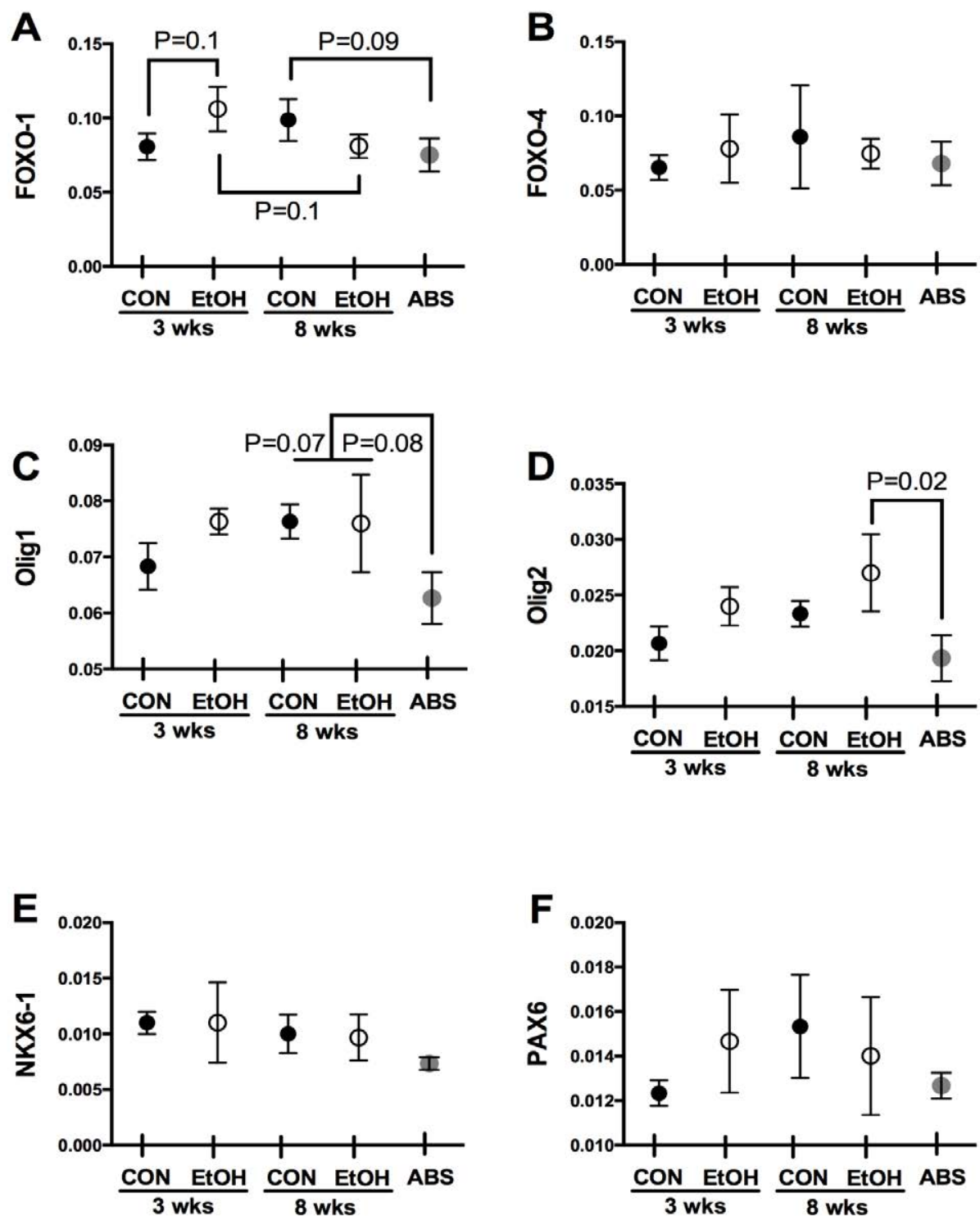

Figure 3: Short- versus long-term ethanol exposure and abbreviated abstinence effects on transcription factor gene expression. mRNA levels of (A) FOXO-1, (B) FOXO-4, (C) Olig1, (D) Olig2, (E) NKX6-1, and (F) PAX6 were measured in temporal lobes of control, ethanol-exposed, and ethanol-abstinent rats by qPCR analysis. Gene expression was calculated by the $2^{-\Delta \Delta C t}$ method and results were normalized to Hypoxanthine-guanine phosphoribosyltransferase (HPRT) and Beta-actin (Act $\beta$ ). Inter-group comparisons were made by one-way and two-way analysis of variance (ANOVA) with Tukey post hoc tests.

and lasted for 12 days. At the endpoints, the animals were either 9 (young adult) or 14 (adult) weeks of age. This design allowed us to study ethanol's detrimental effects on the brain in its late developmental and adult stages and whether these adverse effects could be reversed by short-term abstinence. Histopathological studies revealed that ethanol caused progressive time coursedependent reductions in white matter myelin resembling human alcohol related brain disease $[3,28,38]$. Shortterm abstinence resulted in partial recovery of myelin suggesting similarities with alcohol abstinence effects in alcoholics [10,28,39].

In an earlier study, we demonstrated that longterm chronic + binge ethanol exposures alter ethanol metabolism in young adult rat brains by significantly increasing the activity of alcohol dehydrogenase
$(\mathrm{ADH})$ and aldehyde dehydrogenase (ALDH) in favor of acetaldehyde build-up [40]. Furthermore, alcohol increased oxidative stress marked by increased cellular injury (keratin 18), protein oxidation (protein carbonyl), and lipid peroxidation (8-epimer of Prostaglandin F2 $\alpha$ ) [41]. In contrast to studies using immature (young) rats, the present work showed that chronic + binge ethanol exposures and abbreviated abstinence in adult rats has only modest effects on the expression of immature oligodendrocyte genes. Nestin and GC were the two immature oligodendroglial genes inhibited by ethanol. Nestin is an intermediate filament protein that promotes the disassembly of phosphorylated vimentin during progenitor cell division $[42,43]$ and is necessary for proper survival and self-renewal of neural progenitor cells [44]. GC encodes vitamin D binding protein which promotes proliferation and differentiation of neural stem cells 
into OPCs and oligodendrocytes via vitamin D receptor signaling $[42,45]$. The inhibitory effects of alcohol on nestin and GC suggest that ethanol adversely affects oligodendrocyte survival, maturation and function.

Chronic + binge ethanol exposures had progressive inhibitory effects on the mature oligodendrocyte genes. MAG and RTN4 are potent inhibitors of neurite outgrowth and promote axonal regeneration and plasticity in the adult CNS [46,47]. Ethanol mediated reductions in $\mathrm{MAG}$ mRNA may reflect deficits in glia-axon interactions that are necessary for maintenance and structural integrity of myelinated axons [47]. PLP-1 is the major structural protein of CNS myelin responsible for myelin sheath compaction needed for close apposition of the cytoplasmic and extracellular surfaces of glial plasma membranes [48, 49]. Therefore, the inhibitory effects of alcohol on PLP could compromise the structural and functional integrity of myelin sheaths.

MOG, a minor protein component of myelin, is exclusively expressed on the surface myelin sheath and oligodendrocyte membranes [50]. Since MOG's expression coincides with the onset of myelination, it may represent a marker of oligodendrocyte differentiation and maturation [51]. Correspondingly, our finding that ethanol inhibits MOG may indicate that chronic + binge exposures delay oligodendroglial maturation, which could impact the integrity of myelin homeostasis.

RPA is a single stranded DNA-binding protein involved in DNA replication, repair, and recombination [52]. RPA interacting protein (RPAIN) facilitates import of RPA complex into nucleus and is highly expressed in mature myelinated axons in the CNS [53]. The reductions in RPAIN expression that occurred after a brief interval of abstinence suggest that withdrawal-associated cellular and molecular responses do not rapidly abrogate ethanol's adverse effects on the integrity of CNS myelinated axons and instead may exacerbate pre-existing injury, perhaps due to persistence of metabolic dysfunction.

With respect to neuroglial genes, since NCAM1 mediates neuronal adhesion and neurite outgrowth [34], the elevated expression detected after short-term ethanol exposure likely reflect increased neuritic sprouting responses. However, those effects could be positive and associated with regeneration and repair, or negative and related to oxidative stress. We favor the latter in light of ethanol's simultaneous inhibitory effects on NTRK2 and GSTP1 which respectively function as positive regulator of synapse formation and plasticity [35], and inhibitor of Cdk5 signaling through p25/p35, a driver of neurodegeneration [54].

Transcription factor responses to chronic + binge ethanol exposures varied with duration of exposure and withdrawal, and in ways that could have impaired metabolic, maturation, or homeostatic functions of oligodendrocytes. Ethanol had a stimulatory trend effect on FOXO1 after 3 weeks of exposure, but an inhibitory trend effect after 8 weeks of exposure.FOXO1 transcription factor modulates expression of insulin and insulin-like growth factor regulated target genes involved in glucose and lipid metabolism and favoring states of insulin resistance [36,37,55]. Therefore, with longer durations of ethanol exposure, FOXO1 inhibition may represent a compensatory protective response. The finding that ethanol stimulated Olig2 expression supports the concept that oligodendroglial progenitor cells can be activated in the adult brain [56], perhaps in response to oligodendrocyte death. However, taken together with the impairments in the expression of mature oligodendroglial proteins discussed earlier, the up-regulation of Olig2 would have served to expand a population of immature oligodendroglia that lacked capacity to generate mature compact myelin. The short-term abstinence inhibited of Olig1 and Olig2 expression. The mechanism by which abstinence inhibited Olig1 and Olig2 expression is uncertain, but it may correspond to one of the "switches" that halts proliferation and enables maturation of oligodendroglial cells.

\section{Conclusions}

This study demonstrated that short- and long-term chronic + binge ethanol exposures alter expression of myelin-associated genes and transcription factors such that the aggregate effects are to inhibit maturation and related functions of oligodendrocytes while enabling proliferation of immature oligodendroglia. The net effect would have been to compromise the structural and functional integrity of myelin and myelinated axons in the adult brain. The generally larger effect size associated with longer durations of ethanol exposure reinforce the concept that alcohol-related white matter injury/ degeneration is progressive. The findings with respect to the effects of short-term abstinence were mixed, but at least in several instances, they were consistent with partial recovery responses. Future studies will evaluate recovery responses following longer periods of ethanol abstinence.

\section{References}

1. E. V. Sullivan, M. J. Rosenbloom, and A. Pfefferbaum, Pattern of motor and cognitive deficits in detoxified alcoholic men. Alcoholism, Clinical Exp, 24(2000), 611-621.

2. S. M. de la Monte, and J. J. Kril, Human alcohol-related neuropathology. Acta Neuropathological, 127(2014), 71-90.

3. S. M. de la Monte, Disproportionate atrophy of cerebral white matter in chronic alcoholics, Archives Neurol, 45(1988), 990-992.

4. C. Harper, and J. Kril, Brain atrophy in chronic alcoholic patients: a quantitative pathological study, J Neurology, Neurosurgery, Psychiatry, 48(1985), 211-217.

5. M. J. Rosenbloom, and A. Pfefferbaum, Magnetic resonance imaging of the living brain: evidence for brain degeneration among alcoholics and recovery with abstinence. Alcohol Research \& Health: The J Nat In Alcohol Abuse Alcoholism, 31(2008), 362376 . 
6. A. Papp-Peka, M. Tong, J. J. Kril, S. M. De La Monte, and G. T. Sutherland, The Differential Effects of Alcohol and Nicotine-Specific Nitrosamine Ketone on White Matter Ultrastructure, Alcohol and Alcoholism (Oxford, Oxfordshire), 52(2017), 165-171.

7. T. Schulte, A. Pfefferbaum, and E. V. Sullivan, Parallel interhemispheric processing in aging and alcoholism: relation to corpus callosum size. Neuropsychologia, 42(2004), 257-271.

8. D. Kapogiannis, J. Kisser, C. Davatzikos, L. Ferrucci, J. Metter, and S. M. Resnick, Alcohol consumption and premotor corpus callosum in older adults. European neuropsychopharmacology: the J European College of Neuropsychopharmacology, 22(2012), 704-710.

9. S. Gazdzinski, T. C. Durazzo, A. Mon, P. H. Yeh, and D. J. Meyerhoff, Cerebral white matter recovery in abstinent alcoholics-a multimodality magnetic resonance study. Brain: A J Neurol, 133(2010): 1043-1053.

10. A. J. Bartsch, G. Homola, A. Biller, S. M. Smith, Weijers, H G. Wiesbeck GA, et al., Manifestations of early brain recovery associated with abstinence from alcoholism, Brain :a J Neurol 130(2007), 36-47.

11. M. Kopera, M. Wojnar, K. Brower, J. Glass, I. Nowosad, B. Gmaj, et al., Cognitive functions in abstinent alcohol-dependent patients. Alcohol (Fayetteville, NY), 46(2012), 665-671.

12. G. Bartzokis, P. H. Lu, P. Heydari, A. Couvrette, G. J. Lee, G. Kalashyan, et al., Multimodal magnetic resonance imaging assessment of white matter aging trajectories over the lifespan of healthy individuals. Biol Psy, 72(2012), 1026-1034.

13. K. M. Young, K. Psachoulia, R. B. Tripathi, S. J. Dunn, L. Cossell, D. Attwell, et al., Oligodendrocyte dynamics in the healthy adult CNS: evidence for myelin remodeling, Neuron, 77(2013), 873-885.

14. C. Lebel, L. Walker, A. Leemans, L. Phillips, and C. Beaulieu, Microstructural maturation of the human brain from childhood to adulthood, NeuroImage, 40(2008), 1044-1055.

15. B. D. Semple, K. Blomgren, K. Gimlin, D. M. Ferriero, and L. J. Noble-Haeusslein, Brain development in rodents and humans: Identifying benchmarks of maturation and vulnerability to injury across species, Progress Neurobiol 106-107(2013), 1-16.

16. R. H. Quarles, W. B. Macklin, and P. Morell, Myelin Formation Structure and Biochemistry. 6th ed. Philadelphia: Elsevier; 2006.

17. P. Morell, and R. H. Quarles, Basic Neurochemistry: Molecular, Cellular and Medical Aspects. Characteristic Composition of Myelin. 6th edtn. Philadelphia: Lippincott-Raven; 1999.

18. V. E. Miron, T. Kuhlmann, and J. P. Antel, Cells of the oligodendroglial lineage, myelination, and remyelination, Biochimica et Biophysica Acta, 1812(2011), 184-193.

19. J. J. Boulanger, and C. Messier, From precursors to myelinating oligodendrocytes: contribution of intrinsic and extrinsic factors to white matter plasticity in the adult brain. Neuroscience, 269(2014), 343-366.

20. A. Nishiyama, Polydendrocytes: NG2 cells with many roles in development and repair of the CNS, The Neuroscientist: A Review J Bringing Neurobiology, Neurology and Psychiatry, 13(2007), 62-76.

21. A. Polito, and R. Reynolds, NG2-expressing cells as oligodendrocyte progenitors in the normal and demyelinated adult central nervous system, J Anatomy, 207(2005), 707-716.

22. K. Nixon, and F. T. Crews, Binge ethanol exposure decreases neurogenesis in adult rat hippocampus. J Neurochemistry, 83(2002), 1087-1093.

23. M. Tong, R. Yu, E. Silbermann, V. Zabala, C. Deochand, and S. M. de la Monte, Differential Contributions of Alcohol and NicotineDerived Nitrosamine Ketone (NNK) to White Matter Pathology in the Adolescent Rat Brain. Alcohol and alcoholism (Oxford, Oxfordshire), 50(2015), 680-689.
24. A. Kim, E. R. Zamora-Martinez, S. Edwards, and C. D. Mandyam, Structural reorganization of pyramidal neurons in the medial prefrontal cortex of alcohol dependent rats is associated with altered glial plasticity, Brain Structure \& Function, 220(2015), 1705-1720.

25. A. Pfefferbaum, M. Rosenbloom, T. Rohlfing, and E. V. Sullivan, Degradation of association and projection white matter systems in alcoholism detected with quantitative fiber tracking, Biological Psychiatry, 65(2009), 680-690.

26. F. T. Crews, Alcohol-related neurodegeneration and recovery: mechanisms from animal models. Alcohol research \& health: The $\mathrm{J}$ of the National Institute on Alcohol Abuse and Alcoholism, 31(2008), 377-388.

27. A. I. Navarro, and C. D. Mandyam, Protracted abstinence from chronic ethanol exposure alters the structure of neurons and expression of oligodendrocytes and myelin in the medial prefrontal cortex. Neurosci, 293(2015), 35-44.

28. E. B. Yalcin, T. McLean, M. Tong, and S. M. de la Monte, Progressive white matter atrophy with altered lipid profiles is partially reversed by short-term abstinence in an experimental model of alcohol-related neurodegeneration, Alcohol (Fayetteville, NY), 65(2017), 51-62.

29. K. Nixon, and F. T. Crews, Temporally specific burst in cell proliferation increases hippocampal neurogenesis in protracted abstinence from alcohol. The Journal of neuroscience: the official journal of the Society for Neuroscience, 24(2004), 9714-9722.

30. J. A. Obernier, A. M. White, H. S. Swartzwelder, and F. T. Crews, Cognitive deficits and CNS damage after a 4-day binge ethanol exposure in rats, Pharmacology, Biochemistry, and Behavior, 72(2002), 521-532.

31. M. Ehrlich, S. Mozafari, M. Glatza, L. Starost, S. Velychko, A. L. Hallmann, et al., Rapid and efficient generation of oligodendrocytes from human induced pluripotent stem cells using transcription factors. Proceedings of the National Academy of Sciences of the United States of America, 114(2017), E2243-e2252.

32. M. Watanabe, Y. Sakurai, T. Ichinose, Y. Aikawa, M. Kotani, and K. Itoh, Monoclonal antibody Rip specifically recognizes 2',3'-cyclic nucleotide 3'-phosphodiesterase in oligodendrocytes, $\mathrm{J}$ Neurosci Res, 84(2006), 525-533.

33. T. Kuhlmann, L. Remington, B. Maruschak, T. Owens, and W. Bruck, Nogo-A is a reliable oligodendroglial marker in adult human and mouse CNS and in demyelinated lesions. J Neuropathol Exp Neurol, 66(2007), 238-246.

34. S. D. Skaper, Neuronal growth-promoting and inhibitory cues in neuroprotection and neuroregeneration. Annals of the New York Academy of Sciences, 1053(2005), 376-385.

35. E. J. Huang, and L. F. Reichardt, Trk receptors: roles in neuronal signal transduction. Annual review of biochemistry, 72(2003), 609-642.

36. S. Lee, and H. H. Dong, FoxO integration of insulin signaling with glucose and lipid metabolism, The Journal of endocrinology, 233(2017), R67-R79.

37. A. E. Webb, and A. Brunet, FOXO transcription factors: key regulators of cellular quality control, Trends in Biochem Sci, 39(2014), 159-169.

38. S. M. de la Monte, J. Kay, E. B. Yalcin, J. J. Kril, D. Sheedy, and G. T. Sutherland, Imaging mass spectrometry of frontal white matter lipid changes in human alcoholics, Alcohol (Fayetteville, NY), 67(2018), 51-63.

39. S. C. Bowden, F. T. Crews, M. E. Bates, W. Fals-Stewart, and M. L. Ambrose, Neurotoxicity and neurocognitive impairments with alcohol and drug-use disorders: potential roles in addiction and recovery, Alcoholism, Clinical and Experimental Research, 25(2001), 317-321. 
40. E. B. Yalcin, M. Tong, G. Gallucci, and S. M. de la Monte, Effects of tobacco nicotine-derived nitrosamine ketone (nnk) exposures on brain alcohol metabolizing enzyme activities, Drug Metabolism Lett, 2018.

41. M. Tong, R. Yu, C. Deochand, and S. M. de la Monte, Differential Contributions of Alcohol and the Nicotine-Derived Nitrosamine Ketone (NNK) to Insulin and Insulin-Like Growth Factor Resistance in the Adolescent Rat Brain, Alcohol and alcoholism (Oxford, Oxfordshire), 50(2015), 670-679.

42. G. Almazan, J. M. Vela, E. Molina-Holgado, and C. Guaza, Reevaluation of nestin as a marker of oligodendrocyte lineage cells, Microscopy research and technique, 52(2001), 753-765.

43. Y. H. Chou, S. Khuon, H. Herrmann, and R. D. Goldman, Nestin promotes the phosphorylation-dependent disassembly of vimentin intermediate filaments during mitosis. Molecular Biology of the Cell, 14(2003), 1468-1478.

44. D. Park, A. P. Xiang, F. F. Mao, L. Zhang, C. G. Di, X. M. Liu, et al., Nestin is required for the proper self-renewal of neural stem cells. Stem cells (Dayton, Ohio), 28(2010), 2162-2171.

45. A. G. de la Fuente, O. Errea, P. van Wijngaarden, G. A. Gonzalez, C. Kerninon, A. A. Jarjour, et al., Vitamin D receptor-retinoid $X$ receptor heterodimer signaling regulates oligodendrocyte progenitor cell differentiation, The J Cell Biol, 211(2015), 975-985.

46. M. S. Chen, A. B. Huber, M. E. van der Haar, M. Frank, L. Schnell, A. A. Spillmann, et al., Nogo-A is a myelin-associated neurite outgrowth inhibitor and an antigen for monoclonal antibody IN-1, Nature, 403(2000), 434-439.

47. R. H. Quarles, Myelin-associated glycoprotein (MAG): past, present and beyond, J Neurochemistry, 100(2007), 1431-1448.

48. D. Boison, H. Bussow, D. D'Urso, H. W. Muller, and W. Stoffel, Adhesive properties of proteolipid protein are responsible for the compaction of CNS myelin sheaths, The J Neurosci: The Official J
Society for Neurosci, 15(1995), 5502-5513.

49. V. S. Schwob, H. B. Clark, D. Agrawal, and H. C. Agrawal, Electron microscopic immunocytochemical localization of myelin proteolipid protein and myelin basic protein to oligodendrocytes in rat brain during myelination, J Neurochemistry, 45(1985), 559571.

50. C. Brunner, H. Lassmann, T. V. Waehneldt, J. M. Matthieu, and C. Linington, Differential ultrastructural localization of myelin basic protein, myelin/oligodendroglial glycoprotein, and 2',3'-cyclic nucleotide 3'-phosphodiesterase in the CNS of adult rats. $\mathrm{J}$ Neurochem, 52(1989), 296-304.

51. C. Linington, M. Bradl, H. Lassmann, C. Brunner, and K. Vass, Augmentation of demyelination in rat acute allergic encephalomyelitis by circulating mouse monoclonal antibodies directed against a myelin/oligodendrocyte glycoprotein, The American J Pathol, 130(1988), 443-454.

52. K. Kim, E. J. Lee, S. H. Lee, T. Seo, I. S. Jang, Park J, et al., Speciesspecific variation of RPA-interacting protein (RIP) splice isoforms, BMB Reports, 42(2009), 22-27.

53. B. Friedman, S. Hockfield, J. A. Black, K. A. Woodruff, and S. $\mathrm{G}$.Waxman, In situ demonstration of mature oligodendrocytes and their processes: an immunocytochemical study with a new monoclonal antibody, rip, Glia, 2(1989), 380-390.

54. K. H. Sun, K. H. Chang, S. Clawson, S. Ghosh, H. Mirzaei, F. Regnier, et al., Glutathione-S-transferase P1 is a critical regulator of Cdk5 kinase activity, J Neurochem, 118(2011), 902-914.

55. J. Nakae, M. Oki, and Y. Cao, The FoxO transcription factors and metabolic regulation, FEBS lett, 582(2008), 54-67.

56. K. L. Ligon, S. P. Fancy, R. J. Franklin, and D. H. Rowitch, Olig gene function in CNS development and disease, Glia, 54(2006), $1-10$. 